\title{
Comparative Double Blind Study for the Effect of Dexmedetomidine During Sedation of Obstructive Sleep Apnea Obese Patients for Upper Gastro Endoscopic Procedures
}

\author{
Hany Said ${ }^{1}$, Emad Salem ${ }^{2 *}$, Mohammad A Taha Alafifi ${ }^{\mathbf{1}}$, Amr Allam ${ }^{\mathbf{1}}$ and Mohammad Gaber Saad ${ }^{\mathbf{1}}$ \\ ${ }^{1}$ Department of Anesthesia and Critical Care Medicine, Faculty of Medicine, Alazhar University, Egypt \\ ${ }^{2}$ Department of Gastroenterology and Endoscopy. New Jeddah Clinic Hospital-NJCH-Jeddah, K.S.A \\ *Corresponding Author: Emad Salem, Head of Gastroenterology and Endoscopy Unit at NJCH (New Jeddah Clinic Hospital).
}

Received: June 11, 2019; Published: July 16, 2019

DOI: $10.31080 /$ ASGIS.2019.02.0060

\begin{abstract}
Background and Study Aim: Upper GIT endoscopy became a fundamental method of investigation of the digestive tract, and is important in the diagnosis and prognosis of a variety of gastrointestinal disorders. conventional monosedation usually ineffective if used alone and patient and endoscopist dissatisfaction is common problem. The aim of the present analysis was to evaluate the clinical effect of Dexmedetomidine During Sedation of Obstructive Sleep Apnea Obese Patients For Upper Gastro Endoscopic by adding dexmedetomidine in two groups in combination with another sedative comparing to old conventional group contain combination of midazolam and propofol.

Patients and Methods: 60 Patients were classified according computerized random distribution into 3 groups which were blinded to the endoscopist and observer but well known to the anesthetist each group consists of 20 patients: Group (G1) Patients received midazolam $0.05 \mathrm{mg} / \mathrm{kg}$ then propofol $1-2 \mathrm{mg} / \mathrm{kg}$. Group (G2) Patients received dexametomidine 1 microgram/kg titration over $10 \mathrm{~min}$ then midazolam $0.05 \mathrm{mg} / \mathrm{kg}$ bolus. Group (G3) Patients received dexmetomidine 1 microgram/kg titration over 10 min then propofol 1-2 mg/kg. Intra and post-operative vital signs (pulse-BP-Spo2), assessment of gag reflex and adequacy of breathing, obstruction of the airway, patient satisfaction, endoscopist satisfaction, RSS (Ramsay sedation Scale) and time at which Alderet score > 9 were recorded. End point was $30 \mathrm{~min}$ in recovery room (started immediately after finish of the procedure and patient was shifted to recovery room).

Results: Dexmetomidine in group G3 provide excellent acceptance of endoscopy with very minimal gag as propofol in this group express adequate sedation and cerebral cortex depression beside excellent pain killer effect of dexametomidine due to its central selective $\alpha$-2 agonist effect. It is become more prominent and significant in comparing the mean time from start of induction to reach Aldrete score $>9$ between the studied 3 groups it was short $18.2 \mathrm{~min}$ in group G1, it was $46.2 \mathrm{~min}$ in group G2 and 27 min in group G3, so it is much longer in group G2 (midazolam and dexametomidine). Also G2 and G3 has less respiratory depression and apnea as compared to G1.it reaches to 0\%in both groups G2 and G3 while reach to 15\% in group G1.

Conclusions: The use of Dexmedetomidine can reduce the incidence of respiratory complications and the total dose of other sedative agents. Combined protocol using sedative agents with different pharmacokinetics may minimize the side effects of each. Dexmedetomidine can be used as an alternative to conventional methods in combination with midazolam or propofol for adequate sedation for OSA patients during upper gastro-endscopic procedures.
\end{abstract}

Keywords: GIT Endoscopy; Propofol; Dexametomidine

Citation: Emad Salem., et al. "Comparative Double Blind Study for the Effect of Dexmedetomidine During Sedation of Obstructive Sleep Apnea Obese Patients for Upper Gastro Endoscopic Procedures”. Acta Scientific Gastrointestinal Disorders 2.6 (2019): 24-35. 
Comparative Double Blind Study for the Effect of Dexmedetomidine During Sedation of Obstructive Sleep Apnea Obese Patients for Upper Gastro Endoscopic Procedures

\section{Introduction}

Upper GIT endoscopy became a fundamental method of investigation of the digestive tract, and is important in the diagnosis and prognosis of a variety of gastrointestinal disorders. Endoscopy as a noninvasive modality allows visual examination of the digestive mucosa and lumen and facilitates the procurement of specimens for biopsy, cytology, and culture.

Procedural sedation can provide more comfort for the patient and an easier procedure for the clinician for painful or unpleasant diagnostic or therapeutic procedures. It may be preferred over general anesthesia due to physiological, financial and logistical considerations.

Midazolam is one of the classic sedatives for procedural sedation. While midazolam is thought to cause minimal hemodynamic effects, it does have the potential to cause loss of airway reflexes, respiratory depression, and even apnea [1]. If an effective, reliable and safe sedative could be used in general practice, this would benefit a wide range of patients, especially those who are frail, anxious, severely phobic or uncooperative.

Dexmedetomidine (an alpha2-adrenergic agonist) is a relatively new drug, Dexmedetomidine acts primarily on the locus ceruleus of the pons [2]. It exhibits unique sedative activity not found in conventional sedatives and is thus unlikely to cause the respiratory suppression seen with GABA receptor agonists such as midazolam and propofol.

Dexmedetomidine has sedative and anxiolytic properties and is known for its analgesic potential owing to a reduction of sympathetic tone. Dexmedetomidine induces dose-dependent effects, ranging from minimal to deep sedation. Moreover, except at doses that cause very deep sedation or general anesthesia, the sedation is reversible. The patient can be easily aroused to a lucent state, but when left undisturbed will fall back into a state very similar to natural sleep. These are unique properties among the sedative medications in common use. Dexmedetomidine does not impair the respiratory drive per se and seldom causes apnea. However, it has been shown to impair the respiratory responses to hypoxia and hypercapnia [3] and can cause hemodynamic effects such as hypertension and bradycardia [1].
Many studies have compared aspects of the safety and efficacy of midazolam and dexmedetomidine, but the results have not yet been systematically reviewed. Therefore, the aim of their systematic review was to systematically review the current literature on the relative efficacy and safety of dexmedetomidine and midazolam when used as mono-sedatives for conscious procedural sedation. but they found that they not effective alone for anxious and who request to be in state of moderate to deep sedation to avoid any feel or discomfort during the procedure, so, The objective of our systematic review was to answer the following research question: does dexmedetomidine in combination with midazolam or with propofol or traditional commonly used combination of midazolam and propofol result in more efficacious and safer sedation when used in different upper endoscopic procedures, advantages and disadvantages of each drug combination groups.

\section{Methods}

Study design, data collection and procedures

Our study was conducted on 60 Obese patients in ASA status I-II, aged between 18-60 years old and their BMI more than 35 , considering OSAS (obstructive sleep apnea syndrome) diagnosed by history and clinical suspicion after giving score more than 3 by STOPBANG questionnaire and undergoing upper GIT endoscopy either( gastroscopy - esophageal varices band ligation - gastric balloon insertion- gastric balloon removal) under sedation in endoscopy room ready by anesthesia machine and resuscitation emergency equipment's and crush car in New Jeddah Clinic Hospital (NJCH) -Jeddah.

\section{Exclusion criteria:}

1. Patients Under 18 Years Old or More Than 60 Years Old.

2. Patients with drug or alcohol abusers or those having history of chronic narcotic analgesic use.

3. Patients who known to have allergy against the study drugs.

4. Patients those with 2nd-3rd degree A-V block.

5. Patients with psychiatric disorders.

6. Patients with severe hepatic or renal dysfunction.

Following a fasting for 8 hours, patients were taken to the endoscopy room and a peripheral intravenous cannulation on the dorsal side of the non-dependent hand was performed. A balanced 
crystalloid $150 \mathrm{~mL} / \mathrm{h}$ infusion. Mean arterial pressure (MAP), peripheral oxygen saturation $\left(\mathrm{SpO}_{2}\right)$, heart rate (HR), and Electrocardiogram (ECG) all was connected for the patient.

Patients were classified according computerized random distribution into 3 groups which were blinded to the endoscopist and observer but well known to the anesthetist each group consists of 20 patients:

- Group (G1) Patients received midazolam $0.05 \mathrm{mg} / \mathrm{kg}$ then propofol $1-2 \mathrm{mg} / \mathrm{kg}$ just before procedure start to depress conscious level to greater extent.

- Group (G2) Patients received dexametomidine 1microgram/kg titration over $10 \mathrm{~min}$ then midazolam $0.05 \mathrm{mg} /$ kg bolus administrated just before procedure start to depress conscious level to greater extent.

- Group (G3) Patients received dexametomidine 1microgram $/ \mathrm{kg}$ titration over $10 \mathrm{~min}$ then propofol $1-2 \mathrm{mg} / \mathrm{kg}$ just before procedure start to depress conscious level to greater extent.

Intra and post-operative vital signs (pulse-BP-Spo2), assessment of gag reflex and adequacy of breathing, obstruction of the airway, patient satisfaction, endoscopist satisfaction, RSS (Ramsay sedation Scale) and time at which Alderet score $>9$ were recorded. End point was $30 \mathrm{~min}$ in recovery room (started immediately after finish of the procedure and patient was shifted to recovery room).

All patients received $2 \mathrm{~L}$ /minutes oxygen during the procedure through a nasal cannula. RSS was measured at intervals; at 5, 10, and 15 minutes during the procedure. Time to achieve satisfactory sedation was recorded as minutes (min).

Time to achieve enough sedation was defined as the duration between initiation of the drug infusion and the time when RSS:4. Aldrete recovery scores, hemodynamic and respiratory parameters, RSS and side effects, if occurs, were recorded when the pa- tients arrived at the recovery room (0), and at 10, 20 and 30 minutes. After monitoring $30 \mathrm{~min}$ in the recovery room, patients could be taken to the ward with an Aldrete score $>9$, then monitored in the ward for 4 hours and discharged at the end of this duration. patients' and endoscopist's satisfaction were recorded.

In case of heart rate dropped under 50 beats/minute and continued for 15 seconds in the intra-operative period, it was considered as bradycardia and atropine $0.5 \mathrm{mg}$ iv was administered; similarly in case of MAP dropped by more than $30 \%$ of the initial value in two sequential measures, crystalloid intravenous infusion was raised to $20 \mathrm{ml} /$ minute and hypotension therapy was planned by small IV bolus of Ephedrine 5 - $10 \mathrm{mg}$. Oxygen administration of $5 \mathrm{~L} / \mathrm{min}$ utes with a mask if SPO2 $<92$ due to airway obstruction and head tilt and chin left maneuver or jaw thrust or oropharyngeal airway was inserted. If Apnea or bradypnea happened and desaturation SPO2 < 92 positive pressure ventilation with Ambu bag mask till condition improved and stabilized. If severe unrelieved laryngeal spasm happened or patient vomit during the procedure, tracheal intubation immediately after Suxamethonium chloride intravenous administration.

\section{Results}

Table 1 shows that the highest mean of pulse drop was noticed in group 2 and group $3(43.1 \pm 6.9$ and $23.6 \pm 7.9)$ respectively. It was shown that there was statistical significance difference among the mean pulse drop in the studied groups $(\mathrm{p}<0.05)$. As regards mean drop of systolic blood pressure, it was found that the highest drops were observed in group 3 and group 2 (25.9 \pm 17.6 and 15.1 \pm 10.8 ) respectively with statistical significance difference $(\mathrm{p}<0.05)$ among the studied groups. Regarding SOP2 drop, it was reported that the highest drop was seen in group $1(4 \pm 8.4)$ with no statistical significance difference ( $p>0.05$ ) among the studied groups.

\begin{tabular}{|l|c|c|c|c|c|}
\hline $\begin{array}{l}\text { Operative vital } \\
\text { signs }\end{array}$ & $\begin{array}{c}\text { Group 1 (PM) } \\
\mathbf{N = 2 0}\end{array}$ & $\begin{array}{c}\text { Group 2 (MD)N } \\
\mathbf{2 ~ 2 0}\end{array}$ & $\begin{array}{c}\text { Group 3 (PD) } \\
\mathbf{N = 2 0}\end{array}$ & F TEST & P value \\
\hline Pulse drop & & & & & \\
Rang & $0-20$ & $24-53$ & $8-42$ & & \\
Mean \pm St. D & $5.2 \pm 4.9$ & $43.1 \pm 6.9$ & $23.6 \pm 7.9$ & 94.3 & 0.0 (significant) \\
\hline $\begin{array}{l}\text { Systolic blood } \\
\text { pressure drop }\end{array}$ & $0-20$ & $0-30$ & $3-60$ & & \\
Range & $8.5 \pm 6.9$ & $15.1 \pm 10.8$ & $25.9 \pm 17.6$ & 9.7 & 0.0002 (significant) \\
Mean \pm St. D & & & & & \\
\hline SPO2 drop & $0-30$ & $0-1$ & $0-13$ & & \\
Range & $4 \pm 8.4$ & $0.1 \pm 0.3$ & $1.2 \pm 3.5$ & 2.8 & 0.06 \\
Mean \pm St. D & &
\end{tabular}

Table 1: Operative vital signs among the studied groups. 
Table 2 shows that majority (85\%) of the patients among the studied groups were shown no Gag at the start of endoscopy with no statistical significance difference ( $\mathrm{p}>0.05$ ), also majority $(80 \%)$ of the cases among the studied groups were Airway protected with statistical significance difference $(\mathrm{p}<0.05)$. It was noted that mostly $(85 \%)$ of the examined cases in group 1 were having adequate ventilationwhile all cases in group 2 and 3 were having adequate ventilation. However, there was statistical significance difference $(\mathrm{p}<0.05)$.

\begin{tabular}{|c|c|c|c|}
\hline Patients' signs & $\begin{array}{c}\text { Group } 1 \\
\text { (PM) } \\
N=20\end{array}$ & $\begin{array}{c}\text { Group } 2 \\
\text { (MD) } \\
\mathrm{N}=\mathbf{2 0}\end{array}$ & $\begin{array}{c}\text { Group } 3 \\
\text { (PD) } \\
\mathbf{N}=\mathbf{2 0}\end{array}$ \\
\hline \multicolumn{4}{|l|}{$\begin{array}{l}\text { Gag at start of } \\
\text { endoscopy }\end{array}$} \\
\hline No & $17(85 \%)$ & $16(80 \%)$ & $16(88.9 \%)$ \\
\hline Yes & $3(15 \%)$ & $4(20 \%)$ & $2(11.1 \%)$ \\
\hline Chi2 $=0.1$ & & & \\
\hline & 0 & 0 & $2(11.1)$ \\
\hline $\begin{array}{l}\text { Not applicable (colonos- } \\
\text { copy) }\end{array}$ & & & \\
\hline \multicolumn{4}{|l|}{$\begin{array}{l}\text { Airway Protected/ } \\
\text { intervention }\end{array}$} \\
\hline \multicolumn{4}{|l|}{ Protected } \\
\hline Intervention & $16(80 \%)$ & $20(100 \%)$ & $18(90 \%)$ \\
\hline Chi2 = 12.4 & $4(20 \%)$ & $0(0 \%)$ & $2(10 \%)$ \\
\hline \multicolumn{4}{|l|}{$\begin{array}{l}P \text { value }=0.01 \\
\text { (significant) }\end{array}$} \\
\hline \multirow{2}{*}{\multicolumn{4}{|c|}{$\begin{array}{l}\text { Ventilation Adequacy/ } \\
\text { Apnea } \\
\text { Range }\end{array}$}} \\
\hline & & & \\
\hline Adequate & $17(85 \%)$ & $20(100 \%)$ & $20(100 \%)$ \\
\hline Apnea & $3(15 \%)$ & $0(0 \%)$ & $0(0 \%)$ \\
\hline Chi $2=6.3$ & & & \\
\hline $\begin{array}{l}P \text { value }=0.04 \\
\text { (significant) }\end{array}$ & & & \\
\hline
\end{tabular}

Table 2: Gag at start of endoscopy, Airway Protected/intervention and Ventilation Adequacy/Apnea among the studied groups.
Table 3 shows that nearly almost endoscopists (95\%, 85\%, 95\%) in group 1, 2 and 3 respectively were satisfied. Also, all patients included in the 3 groups were totally (100\%) satisfied.

\begin{tabular}{|l|c|c|c|}
\hline Satisfaction & $\begin{array}{c}\text { Group 1 (PM) } \\
\mathbf{N = 2 0}\end{array}$ & $\begin{array}{c}\text { Group 2 (MD) } \\
\mathbf{N = 2 0}\end{array}$ & $\begin{array}{c}\text { Group 3 (PD) } \\
\mathbf{N = 2 0}\end{array}$ \\
\hline Endoscopist & & & \\
Yes & $19(95 \%)$ & $17(85 \%)$ & $19(95 \%)$ \\
No & $1(5 \%)$ & $3(15 \%)$ & $1(5 \%)$ \\
Chi $2=1.7$ & & & \\
P value =0.4 & & & \\
\hline Patient & & $20(100 \%)$ & $20(100 \%)$ \\
Yes & $20(100 \%)$ & $0(0 \%)$ & $0(0 \%)$ \\
No & $0(0 \%)$ & & \\
\hline
\end{tabular}

Table 3: Endoscopist's satisfaction and Patient's satisfaction among the studied groups.

Table 4 shows that group 2 was the longest one in reaching Aldrete score $>9$. This expressed by Mean was $46.2 \pm 7.7$, median was 45 , mode was 40 and range was 35-70. It was observed that there was statistical significance difference $(\mathrm{p}<0.05)$ between the means of the 3 studied groups.

\begin{tabular}{|c|c|c|c|c|c|}
\hline $\begin{array}{l}\text { Time } \\
\text { reached Al- } \\
\text { drete score } \\
>9\end{array}$ & $\begin{array}{c}\text { Group } 1 \\
\text { (PM) } \\
\mathbf{N}=20\end{array}$ & $\begin{array}{c}\text { Group } 2 \\
\text { (MD) } \\
\mathbf{N}=\mathbf{2 0}\end{array}$ & $\begin{array}{c}\text { Group } 3 \\
\text { (PD) } \\
N=\mathbf{2 0}\end{array}$ & $\begin{array}{c}F \\
\text { test }\end{array}$ & $\begin{array}{c}\text { P } \\
\text { val- } \\
\text { ue }\end{array}$ \\
\hline Range & $10-25$ & $35-70$ & $20-35$ & & \\
\hline Mode & 20 & 40 & 25 & & \\
\hline Median & 20 & 45 & 25 & & \\
\hline Mean \pm St. D. & $18.2 \pm 3.7$ & $46.2 \pm 7.7$ & $27.0 \pm 4.9$ & 124.5 & $\begin{array}{l}0.0 \\
\text { (sig- } \\
\text { nifi- } \\
\text { cant) }\end{array}$ \\
\hline
\end{tabular}

Table 4: Time reached Aldrete Score > 9 among the studied groups. 


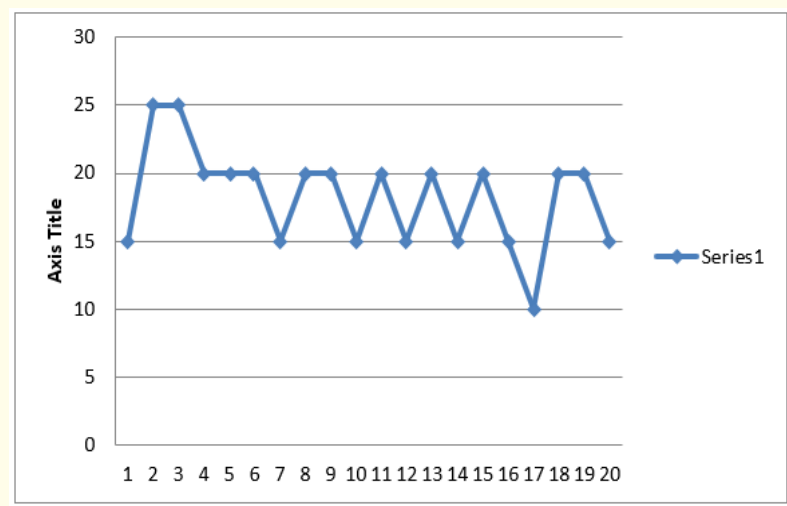

Figure 1: Time reached Aldrete score $>9$ in group 1.

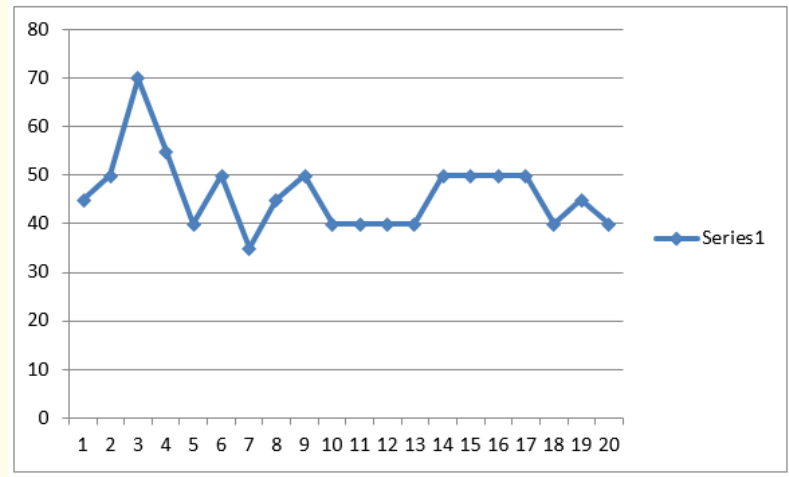

Figure 2: Time reached Aldrete score $>9$ in group 2.

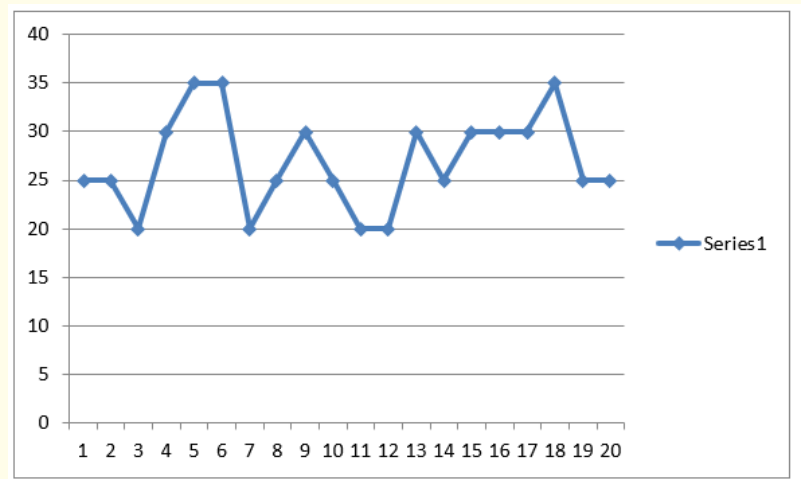

Figure 3: Time reached Aldrete score $>9$ in group 3 .
Table 5 shows the mean of RSS at 15 minutes was the same figure in group 1 and 3 . Also, the range in group 1 and 3 was the same.

\begin{tabular}{|l|c|c|c|}
\hline RSS & $\begin{array}{c}\text { Group 1 (PM) } \\
\text { N = 20 }\end{array}$ & $\begin{array}{c}\text { Group 2 (MD) } \\
\mathbf{~ N ~ = ~ 2 0 ~}\end{array}$ & $\begin{array}{c}\text { Group 3 (PD) } \\
\text { N = 20 }\end{array}$ \\
\hline Range & $4-5$ & - & $4-5$ \\
Mode & 4 & 6 & 4 \\
Median & 4 & 6 & 4 \\
Mean \pm St. D. & $4.4 \pm 0.5$ & - & $4.4 \pm 0.5$ \\
\hline
\end{tabular}

Table 5: RSS at 15 minutes among the studied groups.

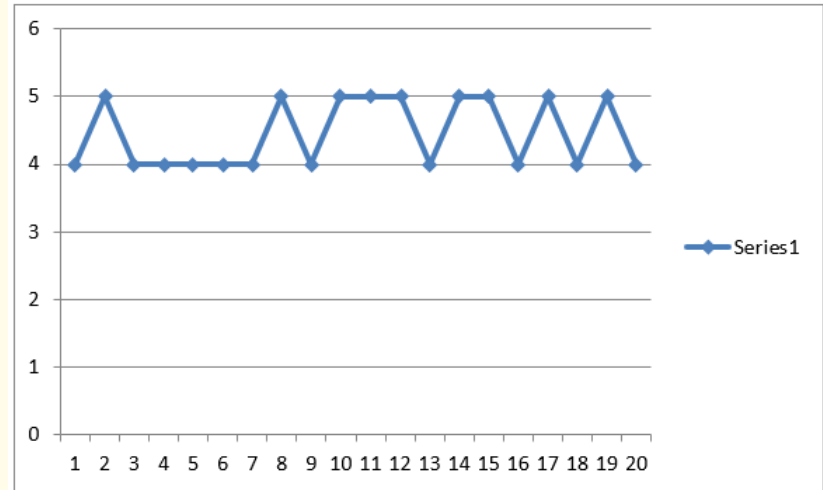

Figure 4: RSS at 15 minutes in group 1.

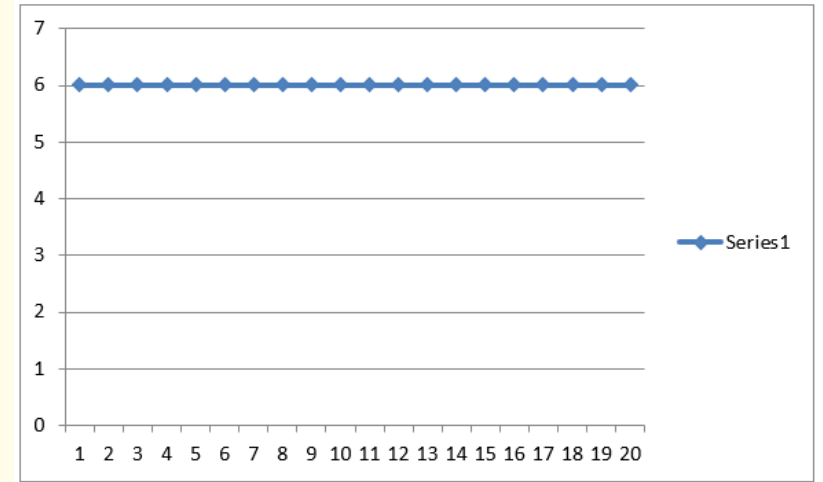

Figure 5: RSS at 15 minutes in group 2. 


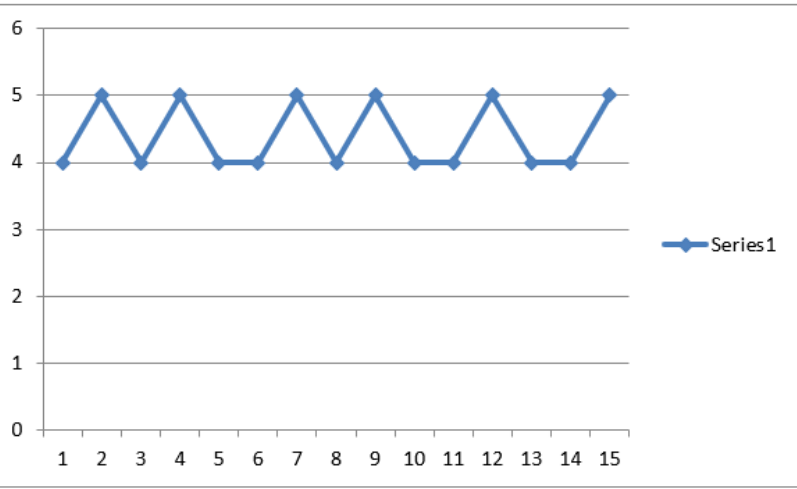

Figure 6: RSS at 15 minutes in group 3.
Table 6 shows that in group 1; 100\% of the examined cases reached score 10 at 30 minutes while no cases were reached score 9 or 10 at 0 minutes. $60 \%$ of cases were reached score 7 at $0 \mathrm{~min}$ utes and $40 \%$ of cases were reached score 8 at 0 minutes.

In group 2; only $10 \%$ of the patients in group 1 reached Adrete score 10 at 30 minutes while $90 \%$ were reached Aldrete score 9 after 30 minutes. It was observed that no cases in group 1 reached score 8,9 or 10 at 0 minutes while $60 \%$ of cases in group 1 reached score 6 at 0 minutes and 35\% of cases reached score 7 . As regards group 3; all cases were reached score 10 at 30 minutes. It was seen that $85 \%$ of cases reached score 10 at 20 minutes and only $15 \%$ reached score 9 at 20 minutes.

It was noticed that there is statistical significance difference between the 3 studied groups at 0,10 and 20 minutes.

\begin{tabular}{|c|c|c|c|c|}
\hline Aldrete score & $\begin{array}{c}\text { Frequency at } \\
\text { (0 minutes) }\end{array}$ & $\begin{array}{l}\text { Frequency at } \\
\text { (10 minutes) }\end{array}$ & $\begin{array}{l}\text { Frequency at } \\
\text { (20 minutes) }\end{array}$ & $\begin{array}{l}\text { Frequency at } \\
\text { (30 minutes) }\end{array}$ \\
\hline \multicolumn{5}{|c|}{ Group $1(N=20)$} \\
\hline Score 7 & $12(60 \%)$ & - & - & - \\
\hline Score 8 & $8(40 \%)$ & $5(25 \%)$ & - & - \\
\hline Score 9 & - & $10(75 \%)$ & $1(5 \%)$ & - \\
\hline Score 10 & - & $5(25 \%)$ & $19(95 \%)$ & $20(100 \%)$ \\
\hline \multicolumn{5}{|c|}{ Group $2(N=20)$} \\
\hline Score 5 & $1(5 \%)$ & - & - & - \\
\hline Score 6 & $12(60 \%)$ & $4(20 \%)$ & - & - \\
\hline Score 7 & $7(35 \%)$ & $11(55 \%)$ & $5(25 \%)$ & - \\
\hline Score 8 & - & $4(20 \%)$ & $11(55 \%)$ & - \\
\hline Score 9 & - & $1(5 \%)$ & $4(20 \%)$ & $18(90 \%)$ \\
\hline Score 10 & - & - & - & $2(10 \%)$ \\
\hline \multicolumn{5}{|c|}{ Group $3(N=20)$} \\
\hline Score 7 & $8(40 \%)$ & - & - & - \\
\hline Score 8 & $8(40 \%)$ & $2(10 \%)$ & - & - \\
\hline Score 9 & $4(20 \%)$ & $12(60 \%)$ & $3(15 \%)$ & - \\
\hline Score 10 & - & $6(30 \%)$ & $17(85 \%)$ & $20(100 \%)$ \\
\hline \multicolumn{5}{|l|}{ Mean \pm St. D. } \\
\hline Group 1 & $7.4 \pm 0.5$ & $9.0 \pm 0.7$ & $9.9 \pm 0.2$ & - \\
\hline Group 2 & $6.3 \pm 0.6$ & $7.1 \pm 0.8$ & $7.9 \pm 0.6$ & $9.1 \pm 0.09$ \\
\hline Group 3 & $7.8 \pm 0.8$ & $9.2 \pm 0.6$ & $9.8 \pm 0.3$ & - \\
\hline F test & 30.9 & 52.8 & 116.3 & 171.0 \\
\hline$P$ value & 0.0 (significant) & 0.0 (significant) & 0.0 (significant) & - \\
\hline
\end{tabular}

Table 6: Frequency of Aldrete score at different times among the studied groups. 


\section{Discussion}

Obstructive Sleep Apnea Syndrome (OSAS) is characterized by the episodes of apnea or hypopnea due to obstruction in the upper airways. If these patients are left untreated, systemic inflammation may develop and cause to several diseases leading to morbidity and mortality such as systemic hypertension, pulmonary hypertension and cor-pulmonal, stroke and metabolic syndrome [1,2].

Several sedative and analgesic drugs are commonly used in GIE procedures the (gastrointestinal endoscopic procedures). Their safety profile is dependent on their pharmacokinetic and pharmacodynamic profiles, the patient medical condition and the experience of the physician using it. From The different anesthetic options most suitable in the endoscopic procedures for anesthetist and endoscopists also the use of most safe sedative agent which will not cause respiratory and cardiac dysfunctions specially in OSA patients which are prone to severe airway obstruction and respiratory depression and may be catastrophic if not ideally managed [4].

Gastrointestinal endoscopy is an uncomfortable and stressful procedure for most patients. Conscious sedation is a common strategy for improving patient comfort during this procedure. Benzodiazepines (gamma-aminobutyric acid (GABA) agonists) such as midazolam have been used for sedation of patients undergoing gastrointestinal endoscopy [5]. The effective dose ranges of such agents differ considerably among patients, making it difficult to achieve stable sedation [6].

Propofol is a powerful sedative that has recently become the "gold standard" for moderate to deep procedural sedation because of its rapid "onset" and "offset" of action modes. Dexmedetomidine is used for light to mild sedation [7]. Patients might prefer propofol administration for its deeper sedation. Dexmedetomidine has a short half-life (two to three hours), whereas propofol has a three times shorter half-life (30-60 minutes) [8].

Propofol, a phenolic derivative, has sedative and hypnotic effects that are mediated by the GABA receptor. It has no analgesic action. It is highly lipophilic, and thus can rapidly cross the bloodbrain barrier, resulting in an early onset of action. The most important disadvantage of propofol is the risk of rapidly induced deep sedation, with the possibility of causing respiratory and cardiovascular depression [9]. On the other hand, recent meta-analysis shows that the use of propofol as a sedative during gastrointestinal endoscopy provides a shorter recovery time and better sedation than traditional sedative agents without causing an increase in cardiopulmonary complications [10].

Dexmedetomidine (Precedex; Hospira Japan Co., Osaka, Japan) is an alpha-2 receptor agonist with sedative, analgesic, and anxiolytic properties [11]. It is a first-line drug for sedation in intensive care units [12]. Recent meta-analysis found that dexmedetomidine for gastrointestinal endoscopy provides better sedation than traditional sedative agents without causing an increase in cardiopulmonary complications [13].

In our study we found that patient receiving dexametomidine either in group G2 or G3 are more liable for bradycardia and hypotension and some of them received atropine and ephedrine more than patients in group G1 who did not received dexametomidine at all and only received midazolam with propofol and this is due to sympatholytic effect of dexametomidine in G2 and G3 groups while dexametomidine provide adequate breathing without drop of Spo2 in both G2 andG3 groups and it seems very helpful in obese patients. Dexmedetomidine is a high selective $\alpha-2$ adrenergic receptor agonist and as a result of the stimulation of these receptors, central sympathetic activity decreases and, sedative and anxiolytic effects reveal [14]. This drug is known to show adrenergic effect with rapid loading. Therefore, loading dose is recommended to be administered slowly at least 10 minutes for the sympatholytic effect. They applied the loading dose for 10 minutes and did not cause occurrence of sympathetic activity. Although dexmedetomidine temporarily increases the blood pressure and heart rate in the beginning, this effect is replaced by drops in the blood pressure and heart rate [15]. Some of the sedo-analgesia studies comparing dexmedetomidine and propofol reported that both drugs decrease MAP and HR.

In our study also, we notice that many patients enter in gag at start of endoscopy around 20\% (4 patients) in group G2, and this give bad impression and increase dissatisfaction of endoscopist in this group up to $15 \%$ compared by $5 \%$ dissatisfaction only in groups G1 and G3.

Our explanation in this, that midazolam in group G2 does not express enough depression of conscious level enough to accept entrance of endoscopy and may need further dose than $0.05 \mathrm{mg} / \mathrm{kg}$. 
While in group G3 we notice excellent acceptance of endoscopy with very minimal gag as propofol in this group express adequate sedation and cerebral cortex depression beside excellent pain killer effect of dexametomidine due to its central selective $\alpha$ - 2 agonist effect (sedo-analgesia).

Combined use of propofol, benzodiazepine, opioids or dexmedetomidine provides more advantages due to the synergistic effect compared to the using of these agents alone $[16,17]$. Muller., et al. [18] has found that dexmedetomidine alone was not as effective as propofol combined with fentanyl for providing conscious sedation.

We administered iv dexmedtomidine prior to midazolam or propofol aiming to take advantage of the synergistic effect and less amount of propofol consumption if used alone that can exceed $800 \mathrm{mg}$ propofol in complicated cases if it is the soul anesthetic during the procedure, and avoid its profound hypotension and take the advantages of less respiratory depression of both midazolam and dexmedetomidine.

Many studies found that BIS values decreased, and sedation score increased more rapidly in the propofol group; thus, time to achieve adequate sedation was shorter in the propofol group. On the other hand, in dexmedetomidine group, we observed adequate sedation as well as respiratory stability.

In our study we found that both group G2 (midazolam and dexmedetomidine) and group G3(propofol and dexmedetomidine) has less respiratory depression and apnea as compared to G1(midazolam and propofol), it reaches to 0\%in both groups G2 and G3 while reach to 15\% in group G1as combination of both midazolam and propofol is stronger than other combination in different groups to develop apnea. propofol might cause hypotonia and depress breathing due to its muscle relaxant effects.

As regard for airway obstruction percent we notice that no patients developed airway obstruction that need airway intervention $0 \%$ in group G2 while increase slightly in group G3 to be only $10 \%$ of the patients became obstructed and need airway intervention either head tilt and chin left or jaw thrust or nasal or oral airway insertion while increase markedly to reach maximum $20 \%$ in groupG1.

Gross., et al. [19] demonstrated that, there is a tendency to decrease in physiological responses against hypoxia and hypercapnia in OSAS and, susceptibility increases against anesthetic agents which have effects causing respiratory depression. Consistent with data from their study, the sedo-analgesia studies comparing propofol and dexmedetomidine have found higher respiratory rate and oxygen saturation in the blood in the dexmedetomidine group and they explained that propofol might cause hypotonia and depress breathing due to its muscle relaxant effects. They reported that dexmedetomidine provided a better respiratory stability and was more attractive drug for anesthetist due to its the wide margin of safety [20-22]. their results support these studies; despite they used low dose propofol with controlled infusion instead of bolus injection, peripheral oxygen saturation and respiratory rates were higher in dexmedetomidine group than propofol group. In addition, the lowest $\mathrm{SpO}_{2}$ value was recorded in propofol group during the procedure and oxygen support was needed.

Sedation in the patients with obstructive sleep apnea (OSA) is very challenge. sedation with Dexmedetomidine offers minimal respiratory depression which is a desirable characteristic in the patients with OSA. An observational study assessed the safety and efficacy of dexmedetomidine/propofol anesthesia for the patients with OSA without endotracheal intubation during Esophagogastroduodenoscopy (EGD) procedure. Twenty patients with high probability of OSA undergoing EGD procedure were enrolled in the study. Dexmedetomidine $1 \mathrm{mcg} / \mathrm{kg}$ bolus was administered over $10 \mathrm{~min}$ followed by propofol boluses. After that, the anesthesia was maintained by using continuous propofol infusion. The result showed transient hypoxemic events occurred in two patients during the EGD procedure. Additionally, transient hypotension was experienced in three patients during the procedure and three patients in the post-anesthesia care unit. After discharge, 16 patients complained of drowsiness, two patients informed dysphoric symptoms and one patient reported of dry mouth. The study concluded that 
the combination of dexmedetomidine and propofol could offer acceptable anesthesia for EGD procedure in the patients with OSA. This combination method provided a substitute to tracheal intubation in these high risk patients [23].

In one study, use of propofol in Drug-induced sleep endoscopy (DISE) was criticized and the authors stated that this drug caused more hypotonia and muscle relaxation, lead to deeper sleep and might cause incorrect evaluations about the obstruction [24]. It was demonstrated in some studies that, patients sedated with dexmedetomidine are more cooperative than those sedated with propofol and that, dexmedetomidine provided a faster recovery period and earlier returning to the consciousness level [25-27]. In another study, time of stay in the recovery room was found similar with dexmedetomidine and propofol [28]. This was supported by our results; in this study Aldrete score at time of the admission to the recovery room were higher in dexmedetomidine group. In contrast, it was reported that, time of stay in the recovery room was longer in patients who received dexmedetomidine, thus it was concluded that the use of this drug as a sedo-analgesic agent should be limited [28,25,29,30].

Generally, the combination regimens are commonly used for invasive procedures. Dexmedetomidine may employ a synergistic effect in the combination with sedo-analgesic drugs. Lee and coworkers evaluated the efficacy and adverse effects of midazolammeperidine-dexmedetomidine (MMD) and midazolam-meperidine (MM) for ERCP procedure in 110 patients. Lower additional and total doses of midazolam were needed in group MMD. Oxygen desaturation and pain scores in group MMD were significantly lesser than in group MM. In addition, the satisfaction scores in group MMD were significantly greater than group MM. The authors recommended that the combination of dexmedetomidine, midazolam and meperidine regimen presented superior sedative efficacy and a greater safety profile during ERCP procedure compared with the combination of midazolam and meperidine regimen [31]. Recently, Mukhopadhyay., et al. [32] assess the safety and efficacy of dexmedetomidine as an add-on for deep sedation in prolonged ERCP procedure. The authors concluded that the addition of dexmedetomidine in sedo-analgesic cocktail increased the safety and efficacy of deep sedation [32].

Although sedation by dexmedetomidine has been reported for its usefulness in other endoscopic procedures, such as upper and lower endoscopy for screening purposes and endoscopic submucosal dissection (ESD), its effectiveness in ERCP is controversial. Muller., et al. and Mazanikov., et al. reported that it was not sufficiently effective when used alone [33,34], while Lee., et al. reported the effectiveness of the combined use of midazolam and pethidine hydrochloride in randomized trials [35]. The reason for this discrepancy is that ERCP is a more invasive procedure than other endoscopic procedures, and it is known that injection of contrast medium into the pancreatic duct and bile duct or mechanical expansion of the papilla, including endoscopic papillary balloon dilation, is painful, while mucosal resection in ESD is usually painless. dexmedetomidine is somewhat weaker as a sedative than benzodiazepine and other sedative drugs [36], and a single use does not provide enough sedative effect for ERCP.

Finally ,we notice perfect rapid recovery time in group G1 and G3 when comparing Aldrete score for all groups once arrival of the patient to RR( 0 minutes), it was higher score 7 in group G1around $60 \%$ of the patients achieves score 7 once arrival to RR while the number or percent of patients achieves the same score 7 was lower around $40 \%$ in group G3.In the contrast in group G2 fewer number and small percent of the patients only $35 \%$ achieve the same score 7 at o minutes in RR.

When comparing time and present of the patients became ready for discharge and they reach adequate cognitive function, it reach maximum in group G1 around 95\% of the patients reach score 10 at 20 minutes in the recovery room and $100 \%$ of patients reach score 10 at 30 minutes, then only lesser percent of the patients in group G3 around $85 \%$ of the patients reach score 10 at 20 minutes and all patients $100 \%$ reach score 10 at 30 minutes.

That is means in both groups G1 and G3 all patients $100 \%$ became ready to discharge from RR to their regular room fully awake and transferring themselves to the stretcher and became fully cooperative after 30 minutes staying in RR due to rapid recovery from propofol included in both groups.

But this advantage (as regard for rapid recovery time) was lost in group G2 (midazolam and dexametomidine) as at 20 minutes staying in RR:

No patients reach score 10 at 20 minutes staying in RR and only $20 \%$ of the patient ( 4 patients) achieve score 9 at 20 minutes staying in RR and became ready for discharge to regular room, and 
while the remaining patients were still sleepy Aldrete score ranging from $7-8$ (16patients) and need longer time to be ready to discharge from RR to regular room.

And G2 at 30 minutes staying in RR: 90\% (18 patients) became fully awake reach score 9and became ready to discharge from RR, while only $10 \%$ ( 2 patients) achieves higher 10 score.

That is become more prominent and significant in comparing the mean time from start of induction to reach Aldrete score $>9$ between the studied 3 groups it was short 18.2 min in group G1, it was $46.2 \mathrm{~min}$ in group G2 and $27 \mathrm{~min}$ in group G3 so it is much longer in group G2 (midazolam and dexametomidine).

\section{Conclusion}

In our opinion if anesthetist is anxious about the airway and possibility of airway obstruction or respiratory depression but not interested about the rapid recovery for these obese patients with OSAS, it is excellent choice to use this combination as group G2 (midazolam and dexametomidine) specially if local anesthesia is used by lidocaine spray $10 \%$ for mouth and lidocaine gel swallowing for oropharynx and esophagus.

Also dexamedetomidine in combination with small dose of propofol seems to be more safer in obese patient with OSAS as it decrease the total consumption of propofol as in our study G3 provide adequate rapid and deeper sedation and rapid recovery and less cough and gag with hemodynamic and respiratory stability for obese patients undergoing different upper endoscopic procedures such as(gastroscopy - esophageal varices band ligation - gastric balloon insertion- gastric balloon removal) with better endoscopist satisfaction compared to any different anesthetic technique. As patients with OSAS have higher risk to develop hypoxia and hypercapnia with increased incidence of morbidity.

The use of Dexmedetomidine can reduce the incidence of respiratory complications and the total dose of other sedative agents. Combined protocol using sedative agents with different pharmacokinetics may minimize the side effects of each. Dexmedetomidine can be used as an alternative to conventional methods in combination with midazolam or propofol for adequate sedation for OSA patients during upper gastro-endscopic procedures.

\section{Bibliography}

1. Benton ML and Friedman NS. "Treatment of obstructive sleep apnea syndrome with nasal positive airway pressure improves golf performance". Journal of Clinical Sleep Medicine 9.12 (2013): 1237-1242.

2. Hayashi Y., et al. "Dexmedetomidine prevents epinephrine-induced arrhythmias through stimulation of central $\alpha 2$ adrenoceptors in halothane-anesthetized dogs". Anesthesiology 75.1 (1991):113-117.

3. Vroegop AV., et al. "Observer variation in drug-induced sleep endoscopy: experienced versus nonexperienced ear, nose and throat surgeons". Sleep 36.6 (2013): 947-953.

4. Hannallah M., et al. "Evaluation of dexmedetomidine/propofol anesthesia during upper gastrointestinal endoscopy in patients with high probability of having obstructive sleep apnea". Anaesthesia, Pain and Intensive Care

5. Kashiwagi K., et al. "Prospective, randomized, placebo-controlled trial evaluating the efficacy and safety of propofol sedation by anesthesiologists and gastroenterologist-led teams using computer-assisted personalized sedation during upper and lower gastrointestinal endoscopy". Digestive Endoscopy 28.6 (2016): 657-664.

6. Wang D., et al. "The use of propofol as a sedative agent in gastrointestinal endoscopy: A meta-analysis". PLoS One 8.1 (2013): e53311-e53311.

7. Clarke GA., et al. "The indications, utilization and safety of gastrointestinal endoscopy in an extremely elderly patient cohort". Endoscopy 33.7 (2001): 580-584.

8. Ross WA. "Premedication for upper gastrointestinal endoscopy”. Gastrointestinal Endoscopy 35.2 (1989): 120-126.

9. Nishizawa T., et al. "Propofol versus traditional sedative agents for endoscopic submucosal dissection". Digestive Endoscopy 26.6 (2014): 701-706.

10. Hashiguchi K., et al. "Dexmedetomidine for sedation during upper gastrointestinal endoscopy". Digestive Endoscopyc 20 (2012): 178-183.

11. Coursin DB and Maccioli GA. "Dexmedetomidine". Current Opinion in Critical Care 7.4 (2001): 221-226. 
12. Barr J., et al. "Clinical practice guidelines for the management of pain, agitation, and delirium in adult patients in the intensive care unit". Critical Care Medicine 41.1 (2013): 263-306.

13. Nishizawa T., et al. "Dexmedetomidine versus midazolam for gastrointestinal endoscopy: A meta-analysis". Digestive Endoscopy 27.1 (2015): 8-15.

14. Mathews AMV., et al. "A case report on the Anesthetic Management of Dexmedetomidine-induced Sleep endoscopy and Transoral Robotic Surgery for the Treatment of Obstructive Sleep Apnoea". Proceedings of Singapore Healthcare (2013): 2.

15. Wan Q., et al. "Effects of Dexmedetomidine combined with Dezocine on cognition function and hippocampal microglia activation of rats". International Journal of Clinical and Experimental Medicine 7.9 (2014): 2787-2792.

16. Akarsu Ayazoğlu T., et al. "Comparison of propofol- based sedation regimens administered during colonoscopy". Revista médica de Chile 141.4 (2013): 477-485.

17. Kaygusuz K., et al. "Comparison of sedation with dexmedetomidine or propofol during shockwave lithotripsy: a randomizedcontrolled trial". Anesthesia and Analgesia 106.1 (2008): 114-119.

18. Muller S., et al. "Clinical efficacy of dexmedetomidine alone is less than propofol for conscious sedation during ERCP”. Gastrointestinal Endoscopy 67.4 (2008): 651-659.

19. Gross JB., et al. "Practice guidelines for the perioperative management of patients with obstructive sleep apnea: a report by the American Society of Anesthesiologists Task Force on Perioperative Management of patients with obstructive sleep apnea." Anesthesiology. 120 (2014): 268-286.

20. Techanivate A., et al. "A Comparison of Dexmedetomidine versus Propofol on Hypotension during Colonoscopy under Sedation". Journal of Anesthesia and Clinical Research 3 (2012): 11.

21. Wu W., et al. "Dexmedetomidine versus midazolam for sedation in upper gastrointestinal endoscopy". Journal of International Medical Research 42.2 (2014): 516-522.

22. Sethi P., et al. "Dexmedetomidine versus midazolam for conscious sedation in endoscopic retrograde cholangiopancreatography: an open-label randomised controlled trial". Indian Journal of Anaesthesia 58.1 (2014): 18-24.
23. Hannallah M., et al. "Evaluation of dexmedetomidine/propofol anesthesia during upper gastrointestinal endoscopy in patients with high probability of having obstructive sleep apnea". Anaesthesia, Pain and Intensive Care 17 (2013): 257-260.

24. Mathews AMV., et al. "A case report on the Anesthetic Management of Dexmedetomidine-induced Sleep endoscopy and Transoral Robotic Surgery fort he Treatment of Obstructive Sleep Apnoea". Proceedings of Singapore Healthcare (2013): 2.

25. Akarsu Ayazoğlu T., et al. "Comparison of propofol- based sedation regimens administered during colonoscopy". Revista médica de Chile 141.4 (2013): 477-485.

26. Techanivate A., et al. "A Comparison of Dexmedetomidine versus Propofol on Hypotension during Colonoscopy under Sedation". Journal of Anesthesia and Clinical Research 3 (2012): 11.

27. Kasuya Y., et al. "The correlation between bispectral index and observational sedation scale in volunteers sedated with dexmedetomidine and propofol". Anesthesia and Analgesia 109.6 (2009): 1811-1815.

28. Vanderveken OM. "Drug-induced sleep endoscopy (DISE) for non-CPAP treatment selection in patients with sleep-disordered breathing". Sleep and Breathing 17.1 (2013): 13-14.

29. Pringle MB and Croft CB. "A grading system for patients with obstructive sleep apnoea-based on sleep nasendoscopy". Clinical Otolaryngology and Allied Sciences 18.6 (1993): 480-484.

30. Zeyneloglu P., et al. "Dexmedetomidine causes prolonged recovery when compared with midazolam/fentanyl combination in outpatient shock wave lithotripsy". European Journal of Anaesthesiology 25.12 (2008): 961-967.

31. Lee BS., et al. "Midazolam with meperidine and dexmedetomidine vs. midazolam with meperidine for sedation during ERCP: prospective, randomized, double-blinded trial". Endoscopy 46.4 (2014): 291-298.

32. Mukhopadhyay S., et al. "The dexmedetomidine augmented sedato-analgesic cocktail: an effective approach for sedation in prolonged endoscopic retrograde cholangiopancreatography". Journal of Anaesthesiology Clinical Pharmacology 31.2 (2015): 201-206.

33. Muller S., et al. "Clinical efficacy of dexmedetomidine alone is less than propofol for conscious sedation during ERCP”. Gastrointestinal Endoscopy 67.4 (2008): 651-659. 
34. Mazanikov M., et al. "Dexmedetomidine impairs success of patient-controlled sedation in alcoholics during ERCP: a randomized, double-blind, placebo-controlled study". Surgical Endoscopy 27.6 (2013): 2163-2168.

35. Lee BS., et al. "Midazolam with meperidine and dexmedetomidine vs. midazolam with meperidine for sedation during ERCP: prospective, randomized, double-blinded trial". Endoscopy 46.4 (2014): 291-298.

36. Hall JE., et al. "Sedative, amnestic, and analgesic properties of small-dose dexmedetomidine infusions". Anesthesia and Analgesia 90.3 (2000): 699-705.

Volume 2 Issue 6 August 2019

(C) All rights are reserved by Emad Salem., et al. 